\title{
Approximate Cubic Lie Derivations
}

\author{
Ajda Fošner ${ }^{1}$ and Maja Fošner ${ }^{2}$ \\ ${ }^{1}$ Faculty of Management, University of Primorska, Cankarjeva 5, 6104 Koper, Slovenia \\ ${ }^{2}$ Faculty of Logistics, University of Maribor, Mariborska Cesta 7, 3000 Celje, Slovenia
}

Correspondence should be addressed to Ajda Fošner; ajda.fosner@fm-kp.si

Received 11 April 2013; Accepted 27 June 2013

Academic Editor: Janusz Brzdek

Copyright (C) 2013 A. Fošner and M. Fošner. This is an open access article distributed under the Creative Commons Attribution License, which permits unrestricted use, distribution, and reproduction in any medium, provided the original work is properly cited.

We study the stability and hyperstability of cubic Lie derivations on normed algebras. At the end, we write some additional observations about our results.

\section{Introduction}

A classical question in the theory of functional equations is under what conditions is it true that a mapping which approximately satisfies a functional equation $\mathscr{E}$ must be somehow close to an exact solution of $\mathscr{E}$ ? We say that a functional equation $\mathscr{E}$ is stable if any approximate solution of $\mathscr{E}$ is near to a true solution of $\mathscr{E}$. The study of stability problem for functional equations is related to a question of Ulam [1] concerning the stability of group homomorphisms. This question was affirmatively answered for Banach spaces by Hyers [2]. Subsequently, the result of Hyers was generalized by Aoki [3] for additive mappings and by Rassias [4] for linear mappings by considering an unbounded Cauchy difference. Rassias' paper has provided a lot of influence in the development of what we now call the Hyers-Ulam-Rassias stability of functional equations. For further information about the topic, we refer the reader to $[5,6]$.

Jun and Kim [7] introduced the following functional equation:

$$
\begin{aligned}
f(2 x+y)+f(2 x-y)= & 2 f(x+y)+2 f(x-y) \\
& +12 f(x),
\end{aligned}
$$

and established a general solution for it. Note that, if we replace $x=y=0$ in (1), we get $f(0)=0$. Therefore, setting $x=0$ in (1), we obtain $f(-y)=-f(y)$. Moreover, writing $y=0$ in (1), we get $f(2 x)=8 f(x)$. Now, it is easy to see that a function $f(x)=\lambda x^{3}$ is a solution of (1), where $\lambda$ is a fixed scalar. Thus, it is natural that (1) is called a cubic functional equation. Moreover, every solution of the cubic functional equation is said to be a cubic mapping. In the last few decades, a number of mathematicians worked on the stability of some type of cubic functional equations (see, e.g., [8-13]).

In [14], the authors investigated the stability of cubic derivations in a connection with the functional equation (1). Recently, Yang et al. [15] studied the stability of cubic $*$-derivations on Banach $*$-algebras and in [16] the authors proved the generalized Hyers-Ulam-Rassias stability of ternary cubic derivations on ternary Fréchet algebras. Motivated by these results, we investigate the stability of cubic Lie derivations. In particular, we show that such derivations can be generated by functions which satisfy some quite natural and simple conditions.

\section{Stability of Cubic Lie Derivations}

Before stating our first theorem, let us recall some basic definitions and known results which we will use in the sequel. Throughout the paper, $\mathscr{A}$ will be a complex normed algebra and $\mathscr{M}$ a Banach $\mathscr{A}$-bimodule. We will use the same symbol $\|\cdot\|$ in order to represent the norms on a normed algebra $\mathscr{A}$ and a normed $\mathscr{A}$-bimodule $\mathscr{M}$. For all $x, y \in \mathscr{A}$, the symbol $[x, y]$ will denote the commutator $x y-y x$. We say that a mapping $f: \mathscr{A} \rightarrow \mathscr{M}$ is cubic homogeneous if $f(\lambda x)=$ $\lambda^{3} f(x)$ for all $x \in \mathscr{A}$ and all scalars $\lambda \in \mathbb{C}$. In the following, $\Lambda$ will stand for the set of all complex units; that is,

$$
\Lambda=\{\lambda \in \mathbb{C}:|\lambda|=1\}
$$


A cubic homogeneous mapping $d: \mathscr{A} \rightarrow \mathscr{M}$ is called a cubic derivation if

$$
d(x y)=d(x) y^{3}+x^{3} d(y)
$$

holds for all $x, y \in \mathscr{A}$. Following this notion, we introduce a cubic Lie derivation as a cubic homogeneous mapping $l$ : $\mathscr{A} \rightarrow \mathscr{M}$ satisfying

$$
l([x, y])=\left[l(x), y^{3}\right]+\left[x^{3}, l(y)\right]
$$

for all $x, y \in \mathscr{A}$.

For a given map $f: \mathscr{A} \rightarrow \mathscr{M}$, we consider

$$
\begin{aligned}
\Delta_{f}^{\lambda}(x, y):= & f(2 \lambda x+\lambda y)+f(2 \lambda x-\lambda y)-2 \lambda^{3} f(x+y) \\
& -2 \lambda^{3} f(x-y)-12 \lambda^{3} f(x),
\end{aligned}
$$

where $x, y \in \mathscr{A}, \lambda \in \mathbb{C}$, and

$$
\begin{aligned}
\Delta_{f}(x, y):= & f([x, y])-\left[f(x), y^{3}\right] \\
& -\left[x^{3}, f(y)\right], \quad x, y \in \mathscr{A} .
\end{aligned}
$$

Moreover, for a function $\varphi: \mathscr{A} \times \mathscr{A} \rightarrow[0, \infty)$ we use the following abbreviation:

$$
\phi(x, y):=\sum_{k=0}^{\infty} \frac{\varphi\left(2^{k} x, 2^{k} y\right)}{8^{k}}, \quad x, y \in \mathscr{A} .
$$

Theorem 1. Suppose that $l: \mathscr{A} \rightarrow \mathscr{M}$ is a mapping for which there exists a function $\varphi: \mathscr{A} \times \mathscr{A} \rightarrow[0, \infty)$ such that

$$
\begin{gathered}
\phi(x, y)<\infty, \quad x, y \in \mathscr{A}, \\
\left\|\Delta_{l}^{\lambda}(x, y)\right\| \leq \varphi(x, y), \quad\left\|\Delta_{l}(x, y)\right\| \leq \varphi(x, y)
\end{gathered}
$$

for all $x, y \in \mathscr{A}$ and $\lambda \in \Lambda$. If for each fixed $x \in \mathscr{A}$ the mapping $r \mapsto l(r x)$ from $\mathbb{R}$ to $\mathscr{M}$ is continuous, then there exists a unique cubic Lie derivation $L: \mathscr{A} \rightarrow \mathscr{M}$ such that

$$
\|l(x)-L(x)\| \leq \frac{\phi(x, 0)}{16}
$$

for all $x \in \mathscr{A}$.

Proof. Writing $y=0$ and $\lambda=1$ in the first inequality of (9), we obtain

$$
\left\|\frac{l(2 x)}{8}-l(x)\right\| \leq \frac{\varphi(x, 0)}{16}
$$

for all $x \in \mathscr{A}$. Using the induction, it is easy to see that

$$
\left\|\frac{l\left(2^{p} x\right)}{8^{p}}-\frac{l\left(2^{q} x\right)}{8^{q}}\right\| \leq \frac{1}{16} \sum_{k=q}^{p-1} \frac{\varphi\left(2^{k} x, 0\right)}{8^{k}}
$$

for all $x \in \mathscr{A}$ and all $p>q \geq 0$. By (8), it follows that, for all $x \in \mathscr{A}$, the sequence $\left\{l\left(2^{n} x\right) / 8^{n}\right\}_{n=0}^{\infty}$ is Cauchy and, since $\mathscr{M}$ is complete, it is convergent. Thus, we can define a mapping $L: \mathscr{A} \rightarrow \mathscr{M}$ as

$$
L(x)=\lim _{n \rightarrow \infty} \frac{l\left(2^{n} x\right)}{8^{n}}, \quad x \in \mathscr{A} .
$$

Writing $p=n$ and $q=0$ in (12), we have

$$
\left\|\frac{l\left(2^{n} x\right)}{8^{n}}-l(x)\right\| \leq \frac{1}{16} \sum_{k=0}^{n-1} \frac{\varphi\left(2^{k} x, 0\right)}{8^{k}}
$$

for $n>0$. Taking the limit as $n$ tends to $\infty$ we get (10).

In the following, we will show that $L$ is a unique cubic Lie derivation such that (10) holds true for all $x \in \mathscr{A}$.

Claim 1 ( $L$ is a cubic mapping). Recall that

$$
\begin{aligned}
\left\|\Delta_{L}^{\lambda}(x, y)\right\| & =\lim _{n \rightarrow \infty}\left\|\frac{\Delta_{l}^{\lambda}\left(2^{n} x, 2^{n} y\right)}{8^{n}}\right\| \\
& \leq \lim _{n \rightarrow \infty} \frac{\varphi\left(2^{n} x, 2^{n} y\right)}{8^{n}}=0
\end{aligned}
$$

for all $x, y \in \mathscr{A}$ and $\lambda \in \Lambda$. Hence, taking $\lambda=1$ in (15), it follows that $L$ is a cubic mapping.

Claim 2 (L is cubic homogeneous). By (15), we have $\Delta_{L}^{\lambda}(x, 0)=$ 0 . Thus,

$$
L(2 \lambda x)=8 \lambda^{3} L(x), \quad x \in \mathscr{A}, \lambda \in \Lambda .
$$

Let $x_{0} \in \mathscr{A}$ be any fixed element. Firstly, we will show that $L\left(r x_{0}\right)=r^{3} L\left(x_{0}\right)$ for all $r \in \mathbb{R}$. We will omit the details since the arguments are the same as in [17] (see also [18]). So, let us choose any linear functional $\rho \in \mathscr{M}^{*}$, where $\mathscr{M}^{*}$ denotes the dual space of $\mathscr{M}$. Then, we can define a function $\psi: \mathbb{R} \rightarrow \mathbb{R}$ by

$$
\psi(r)=\rho\left(L\left(r x_{0}\right)\right), \quad r \in \mathbb{R} .
$$

It is easy to see that $\psi$ is cubic. Set

$$
\psi_{n}(r)=\rho\left(\frac{l\left(2^{n} r x_{0}\right)}{8^{n}}\right), \quad n \geq 0 .
$$

Note that $\psi$ is a pointwise limit of a sequence of continuous functions $\psi_{n}, n \geq 0$. Thus, $\psi$ is a continuous cubic function and

$$
\psi(r)=r^{3} \psi(1), \quad r \in \mathbb{R} .
$$

Consequently,

$$
\begin{aligned}
\rho\left(L\left(r x_{0}\right)\right) & =\psi(r)=r^{3} \psi(1)=r^{3} \rho\left(L\left(x_{0}\right)\right) \\
& =\rho\left(r^{3} L\left(x_{0}\right)\right)
\end{aligned}
$$

for all $r \in \mathbb{R}$. Since $\rho \in \mathscr{M}^{*}$ was an arbitrary linear functional, we conclude that

$$
L\left(r x_{0}\right)=r^{3} L\left(x_{0}\right), \quad r \in \mathbb{R} .
$$


Let $\lambda \in \mathbb{C}$. Then $\lambda /|\lambda| \in \Lambda,|\lambda| / 2 \in \mathbb{R}$, and we have

$$
\begin{aligned}
L\left(\lambda x_{0}\right) & =L\left(2 \frac{\lambda}{|\lambda|}\left(\frac{|\lambda|}{2} x_{0}\right)\right)=8\left(\frac{\lambda}{|\lambda|}\right)^{3} L\left(\frac{|\lambda|}{2} x_{0}\right) \\
& =8 \frac{\lambda^{3}}{|\lambda|^{3}}\left(\frac{|\lambda|}{2}\right)^{3} L\left(x_{0}\right)=\lambda^{3} L\left(x_{0}\right) .
\end{aligned}
$$

Since $x_{0}$ was any element from $\mathscr{A}$, we conclude that $L$ is cubic homogeneous.

Claim 3 ( $L$ is a cubic Lie derivation). Using the second inequality in (9), we have

$$
\begin{aligned}
\left\|\Delta_{L}(x, y)\right\| & =\lim _{n \rightarrow \infty}\left\|\frac{\Delta_{l}\left(2^{n} x, 2^{n} y\right)}{8^{2 n}}\right\| \\
& \leq \lim _{n \rightarrow \infty} \frac{\varphi\left(2^{n} x, 2^{n} y\right)}{8^{2 n}}=0
\end{aligned}
$$

for all $x, y \in \mathscr{A}$. This, together with Claim 2, yields that $L$ is a cubic Lie derivation.

Claim 4 ( $L$ is unique). Suppose that there exists another cubic Lie derivation $\widetilde{L}: \mathscr{A} \rightarrow \mathscr{M}$ such that

$$
\|l(x)-\widetilde{L}(x)\| \leq \frac{\phi(x, 0)}{16}
$$

for all $x \in \mathscr{A}$. Then

$$
\begin{aligned}
\|L(x)-\widetilde{L}(x)\| & =\lim _{n \rightarrow \infty} \frac{1}{8^{n}}\left\|l\left(2^{n} x\right)-\widetilde{L}\left(2^{n} x\right)\right\| \\
& \leq \lim _{n \rightarrow \infty} \frac{1}{16}\left(\frac{\phi\left(2^{n} x, 0\right)}{8^{n}}\right) \\
& =\lim _{n \rightarrow \infty} \frac{1}{16} \sum_{k=0}^{\infty} \frac{\varphi\left(2^{k+n} x, 0\right)}{8^{k+n}} \\
& =\lim _{n \rightarrow \infty} \frac{1}{16} \sum_{k=n}^{\infty} \frac{\varphi\left(2^{k} x, 0\right)}{8^{k}}=0 .
\end{aligned}
$$

Therefore, $L(x)=\widetilde{L}(x)$ for all $x \in \mathscr{A}$, which proves the uniqueness of $L$. The proof is completed.

Let $\nu, \varepsilon \geq 0$ and $0 \leq p<3$. Applying Theorem 1 for the case

$$
\varphi(x, y):=v+\varepsilon\left(\|x\|^{p}+\|y\|^{p}\right), \quad x, y \in \mathscr{A},
$$

we have the next corollary.

Corollary 2. Suppose that $l: \mathscr{A} \rightarrow \mathscr{M}$ is a mapping such that (9) holds true for all $x, y \in \mathscr{A}$ and $\lambda \in \Lambda$, where $\varphi$ is a function defined as above. If for each fixed $x \in \mathscr{A}$ the mapping $r \mapsto l(r x)$ from $\mathbb{R}$ to $\mathscr{M}$ is continuous, then there exists a unique cubic Lie derivation $L: \mathscr{A} \rightarrow \mathscr{M}$ such that

$$
\|l(x)-L(x)\| \leq \frac{v}{14}+\frac{\varepsilon\|x\|^{p}}{\left(16-2^{p+1}\right)}
$$

for all $x \in \mathscr{A}$.
Proof. Note that we have

$$
\phi(x, y)=\frac{8 v}{7}+\frac{8 \varepsilon\left(\|x\|^{p}+\|y\|^{p}\right)}{\left(8-2^{p}\right)}, \quad x, y \in \mathscr{A} \text {. }
$$

\section{Hyperstability of Cubic Lie Derivations}

The investigation of the multiplicative Cauchy functional equation highlighted a new phenomenon, which is nowadays called superstability (see, e.g., [19]). In this case the socalled stability inequality implies that the observed function is either bounded or it is a solution of the functional equation. But it can also happen that each function $f$, satisfying the functional equation $\mathscr{E}$ approximately, must actually be a solution of the proposed equation $\mathscr{E}$. In this case, we say that the functional equation $\mathscr{E}$ is hyperstable. According to our best knowledge, the first hyperstability result was published in [20] and concerned the ring homomorphisms. However, the term hyperstability has been used for the first time probably in [21] (see also [22, 23]). For more information about the new results on the hyperstability, we refer the reader to [24-26].

Theorem 3. Let $\mathscr{A}$ be a normed algebra with an element $e$ which is not a zero divisor. Suppose that $l: \mathscr{A} \rightarrow \mathscr{A}$ is a mapping for which there exists a function $\varphi: \mathscr{A} \times \mathscr{A} \rightarrow[0, \infty)$ such that

$$
\begin{gathered}
\lim _{n \rightarrow \infty} \frac{\varphi(n x, y)}{n^{3}}=0 \\
\left\|l(x) y^{3}-x^{3} l(y)\right\| \leq \varphi(x, y) \\
\left\|l(z)([x, y])^{3}-z^{3}\left(\left[l(x), y^{3}\right]+\left[x^{3}, l(y)\right]\right)\right\| \leq \varphi(z,[x, y])
\end{gathered}
$$

for all $x, y, z \in \mathscr{A}$. Then, $l$ is a cubic Lie derivation on $\mathscr{A}$.

Proof. We divide the proof into several steps.

Claim 1 (l is a cubic mapping). Let $x, y, z \in \mathscr{A}$. Then,

$$
\begin{aligned}
\| n^{3} z^{3}(l(2 x+y)+l(2 x-y)-2 l(x+y) \\
\quad-2 l(x-y)-12 l(x)) \| \\
\leq\left\|n^{3} z^{3} l(2 x+y)-l(n z)(2 x+y)^{3}\right\| \\
\quad+\left\|n^{3} z^{3} l(2 x-y)-l(n z)(2 x-y)^{3}\right\| \\
\quad+2\left\|l(n z)(x+y)^{3}-n^{3} z^{3} l(x+y)\right\| \\
\quad+2\left\|l(n z)(x-y)^{3}-n^{3} z^{3} l(x-y)\right\| \\
\quad+12\left\|l(n z) x^{3}-n^{3} z^{3} l(x)\right\|
\end{aligned}
$$


for all positive integers $n$. By (30), it follows that

$$
\begin{aligned}
\| z^{3}( & l(2 x+y)+l(2 x-y)-2 l(x+y) \\
& -2 l(x-y)-12 l(x)) \| \\
\leq & n^{-3}(\varphi(n z, 2 x+y)+\varphi(n z, 2 x-y) \\
& +2 \varphi(n z, x+y)+2 \varphi(n z, x-y) \\
& +12 \varphi(n z, x)) .
\end{aligned}
$$

If we let $n \rightarrow \infty$, we conclude that

$$
\begin{gathered}
z^{3}(l(2 x+y)+l(2 x-y)-2 l(x+y) \\
-2 l(x-y)-12 l(x))=0
\end{gathered}
$$

for all $x, y, z \in \mathscr{A}$. Putting $z=e$ in the last equality, we conclude that $l$ satisfies a cubic functional equation (1).

Claim 2 ( $l$ is cubic homogeneous). Let $\lambda \in \mathbb{C}$ and $x, y \in \mathscr{A}$. Then,

$$
\begin{aligned}
\left\|n^{3} y^{3}\left(l(\lambda x)-\lambda^{3} l(x)\right)\right\| \leq & \left\|n^{3} y^{3} l(\lambda x)-l(n y) \lambda^{3} x^{3}\right\| \\
& +|\lambda|^{3}\left\|l(n y) x^{3}-n^{3} y^{3} l(x)\right\| \\
\leq & \varphi(n y, \lambda x)+|\lambda|^{3} \varphi(n y, x)
\end{aligned}
$$

for all positive integers $n$. Thus,

$$
\left\|y^{3}\left(l(\lambda x)-\lambda^{3} l(x)\right)\right\| \leq n^{-3}\left(\varphi(n y, \lambda x)+|\lambda|^{3} \varphi(n y, x)\right) .
$$

Taking the limit when $n$ tends to $\infty$, we conclude that

$$
y^{3}\left(l(\lambda x)-\lambda^{3} l(x)\right)=0, \quad x, y \in \mathscr{A}, \lambda \in \mathbb{C} .
$$

Writing $y=e$, we get $l(\lambda x)=\lambda^{3} l(x)$ for all $x \in \mathscr{A}$ and $\lambda \in \mathbb{C}$. In other words, $l$ is cubic homogeneous.

Claim 3 ( $l$ is a cubic Lie derivation on $\mathscr{A})$. Let $x, y, z \in \mathscr{A}$. Then

$$
\begin{aligned}
\| n^{3} z^{3} & \left(l([x, y])-\left[l(x), y^{3}\right]-\left[x^{3}, l(y)\right]\right) \| \\
\leq & \left\|n^{3} z^{3} l([x, y])-l(n z)([x, y])^{3}\right\| \\
\quad & +\left\|l(n z)([x, y])^{3}-n^{3} z^{3}\left(\left[l(x), y^{3}\right]+\left[x^{3}, l(y)\right]\right)\right\| \\
\leq & 2 \varphi(n z,[x, y])
\end{aligned}
$$

for all positive integers $n$. Thus,

$$
\begin{aligned}
& \left\|z^{3}\left(l([x, y])-\left[l(x), y^{3}\right]-\left[x^{3}, l(y)\right]\right)\right\| \\
& \quad \leq n^{-3} 2 \varphi(n z,[x, y])
\end{aligned}
$$

for all $x, y, z \in A$ and $n \in \mathbb{N}$. Similarly as in Claims 1 and 2, we can show that $l([x, y])=\left[l(x), y^{3}\right]+\left[x^{3}, l(y)\right]$ for all $x, y \in \mathscr{A}$. The proof is completed.
Let $\varepsilon \geq 0$ and $0 \leq p<3$. Applying Theorem 3 for the case

$$
\varphi(x, y):=\varepsilon\|x\|^{p}\|y\|^{p}, \quad x, y \in \mathscr{A}
$$

we have the next corollary.

Corollary 4. Let $\mathscr{A}$ be a normed algebra with an element $e$ which is not a zero divisor. Suppose that $l: \mathscr{A} \rightarrow \mathscr{A}$ is a mapping such that

$$
\begin{gathered}
\left\|l(x) y^{3}-x^{3} l(y)\right\| \leq \varepsilon\|x\|^{p}\|y\|^{p}, \\
\left\|l(z)[x, y]^{3}-z^{3}\left(\left[l(x), y^{3}\right]+\left[x^{3}, l(y)\right]\right)\right\| \\
\leq \varepsilon\|z\|^{p}\|[x, y]\|^{p}
\end{gathered}
$$

for all $x, y, z \in \mathscr{A}$. Then $l$ is a cubic Lie derivation on $\mathscr{A}$.

\section{Some Additional Remarks}

In this section, we will write some additional observations about our results. We start with the theorem which is analogue to Theorem 1 . Since the proof is similar, it is omitted.

Theorem 5. Suppose that $l: \mathscr{A} \rightarrow \mathscr{M}$ is a mapping for which there exists a function $\varphi: \mathscr{A} \times \mathscr{A} \rightarrow[0, \infty)$ such that (9) holds true for all $x, y \in \mathscr{A}, \lambda \in \Lambda$, and

$$
\sum_{k=0}^{\infty} 8^{k} \varphi\left(\frac{x}{2^{k}}, \frac{y}{2^{k}}\right)<\infty, \quad x, y \in \mathscr{A} .
$$

If for each fixed $x \in \mathscr{A}$ the mapping $r \mapsto l(r x)$ from $\mathbb{R}$ to $\mathscr{M}$ is continuous, then there exists a unique cubic Lie derivation $L: \mathscr{A} \rightarrow \mathscr{M}$ such that (10) is valid for all $x \in \mathscr{A}$.

Similarly, the next result is analogue to Theorem 3.

Theorem 6. Let $\mathscr{A}$ be a normed algebra with an element $e$ which is not a zero divisor. Suppose that $l: \mathscr{A} \rightarrow \mathscr{A}$ is a mapping for which there exists a function $\varphi: \mathscr{A} \times \mathscr{A} \rightarrow[0, \infty)$ such that (30) and (31) hold true for all $x, y, z \in \mathscr{A}$ and

$$
\lim _{n \rightarrow \infty} n^{3} \varphi\left(\frac{x}{n}, y\right)=0, \quad x, y \in \mathscr{A} \text {. }
$$

Then, $l$ is a cubic Lie derivation on $\mathscr{A}$.

Let us end this paper with a remark on $k$-cubic functional equations. In [27], Najati introduced the following functional equations:

$$
\begin{aligned}
f(k x+y)+f(k x-y)= & k f(x+y)+k f(x-y) \\
& +2\left(k^{3}-k\right) f(x),
\end{aligned}
$$

where $k \geq 2$ is a positive integer. For $k=2$, we obtain (1). Najati proved that a function $f$ satisfies the functional equation (1) if and only if $f$ satisfies the functional equation (44). Therefore, every solution of the functional equation (44) is also a cubic function. Following these results, it is easy to see that we can consider (44) in our theorems instead of (1). 


\section{Acknowledgment}

The authors would like to thank the referees for their useful comments.

\section{References}

[1] S. M. Ulam, Problems in Modern Mathematics, John Wiley \& Sons, New York, NY, USA, 1964.

[2] D. H. Hyers, "On the stability of the linear functional equation," Proceedings of the National Academy of Sciences of the United States of America, vol. 27, pp. 222-224, 1941.

[3] T. Aoki, "On the stability of the linear transformation in Banach spaces," Journal of the Mathematical Society of Japan, vol. 2, pp. 64-66, 1950.

[4] T. M. Rassias, "On the stability of the linear mapping in Banach spaces," Proceedings of the American Mathematical Society, vol. 72, no. 2, pp. 297-300, 1978.

[5] D. H. Hyers, G. Isac, and T. M. Rassias, Stability of Functional Equations in Several Variables, Birkhäuser, Boston, Mass, USA, 1998.

[6] S.-M. Jung, Hyers-Ulam-Rassias Stability of Functional Equations in Nonlinear Analysis, vol. 48 of Springer Optimization and Its Applications, Springer, New York, NY, USA, 2011.

[7] K.-W. Jun and H.-M. Kim, "The generalized Hyers-UlamRassias stability of a cubic functional equation," Journal of Mathematical Analysis and Applications, vol. 274, no. 2, pp. 267278, 2002.

[8] A. Bodaghi, I. A. Alias, and M. H. Ghahramani, "Approximately cubic functional equations and cubic multipliers," Journal of Inequalities and Applications, vol. 2011, articlie 53, 2011.

[9] M. E. Gordji, S. K. Gharetapeh, J. M. Rassias, and S. Zolfaghari, "Solution and stability of a mixed type additive, quadratic, and cubic functional equation," Advances in Difference Equations, vol. 2009, pp. 1-17, 2009.

[10] M. Eshaghi Gordji, S. Zolfaghari, J. M. Rassias, and M. B. Savadkouhi, "Solution and stability of a mixed type cubic and quartic functional equation in quasi-Banach spaces," Abstract and Applied Analysis, vol. 2009, Article ID 417473, 14 pages, 2009.

[11] K.-W. Jun, H.-M. Kim, and I.-S. Chang, "On the Hyers-Ulam stability of an Euler-Lagrange type cubic functional equation," Journal of Computational Analysis and Applications, vol. 7, no. 1, pp. 21-33, 2005.

[12] A. Najati, "Hyers-Ulam-Rassias stability of a cubic functional equation," Bulletin of the Korean Mathematical Society, vol. 44, no. 4, pp. 825-840, 2007.

[13] T. Z. Xu, J. M. Rassias, and W. X. Xu, "A generalized mixed type of quartic-cubic-quadratic-additive functional equations," Ukrainian Mathematical Journal, vol. 63, no. 3, pp. 461-479, 2011.

[14] M. Eshaghi Gordji, S. Kaboli Gharetapeh, M. B. Savadkouhi, M. Aghaei, and T. Karimi, "On cubic derivations," International Journal of Mathematical Analysis, vol. 4, no. 49-52, pp. 25012514, 2010.

[15] S. Y. Yang, A. Bodaghi, and K. A. M. Atan, "Approximate cubic *-derivations on Banach *-algebras," Abstract and Applied Analysis, vol. 2012, Article ID 684179, 12 pages, 2012.

[16] B. Hayati, M. E. Gordji, M. B. Savadkouhi, and M. Bidkham, "Stability of ternary cubic derivations on ternary Frèchet algebras," Australian Journal of Basic and Applied Sciences, vol. 5, pp. 1224-1235, 2011.
[17] St. Czerwik, "On the stability of the quadratic mapping in normed spaces," Abhandlungen aus dem Mathematischen Seminar der Universität Hamburg, vol. 62, pp. 59-64, 1992.

[18] S. Kurepa, "On the quadratic functional," Publications de l'Institut Mathématique de l'Académie Serbe des. Sciences, vol. 13, pp. 57-72, 1961.

[19] Z. Moszner, "Sur les définitions différentes de la stabilité des équations fonctionnelles," Aequationes Mathematicae, vol. 68, no. 3, pp. 260-274, 2004.

[20] D. G. Bourgin, "Approximately isometric and multiplicative transformations on continuous function rings," Duke Mathematical Journal, vol. 16, pp. 385-397, 1949.

[21] G. Maksa and Z. Páles, "Hyperstability of a class of linear functional equations," Acta Mathematica. Academiae Paedagogicae Nyíregyháziensis, vol. 17, no. 2, pp. 107-112, 2001.

[22] E. Gselmann, "Hyperstability of a functional equation," Acta Mathematica Hungarica, vol. 124, no. 1-2, pp. 179-188, 2009.

[23] Gy. Maksa, K. Nikodem, and Zs. Páles, "Results on $t$-Wright convexity," L'Académie des Sciences. Comptes Rendus Mathématiques, vol. 13, no. 6, pp. 274-278, 1991.

[24] N. Brillouët-Belluot, J. Brzdęk, and K. Ciepliński, "On some recent developments in Ulam's type stability," Abstract and Applied Analysis, vol. 2012, Article ID 716936, 41 pages, 2012.

[25] J. Brzdęk, "Hyperstability of the Cauchy equation on restricted domains," Acta Mathematica Hungarica, 2013.

[26] M. Piszczek, "Remark on hyperstability of the general linear equation," Aequationes Mathematicae, 2013.

[27] A. Najati, "The generalized Hyers-Ulam-Rassias stability of a cubic functional equation," Turkish Journal of Mathematics, vol. 31, no. 4, pp. 395-408, 2007. 


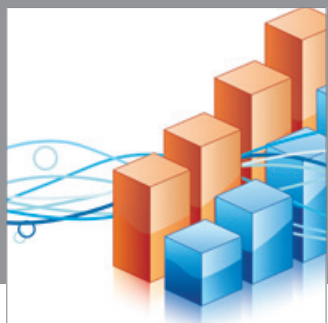

Advances in

Operations Research

mansans

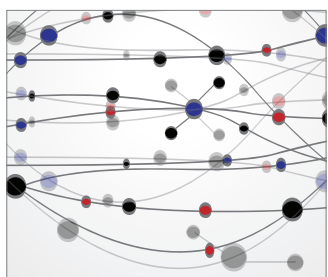

The Scientific World Journal
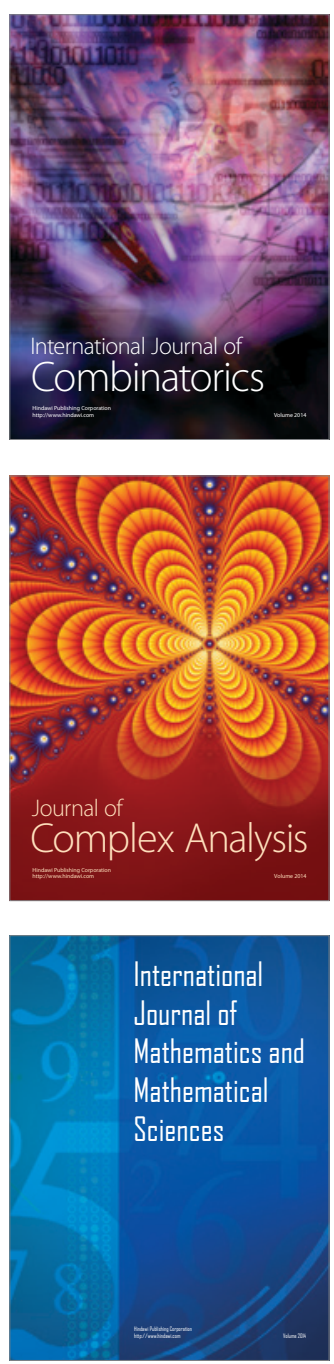
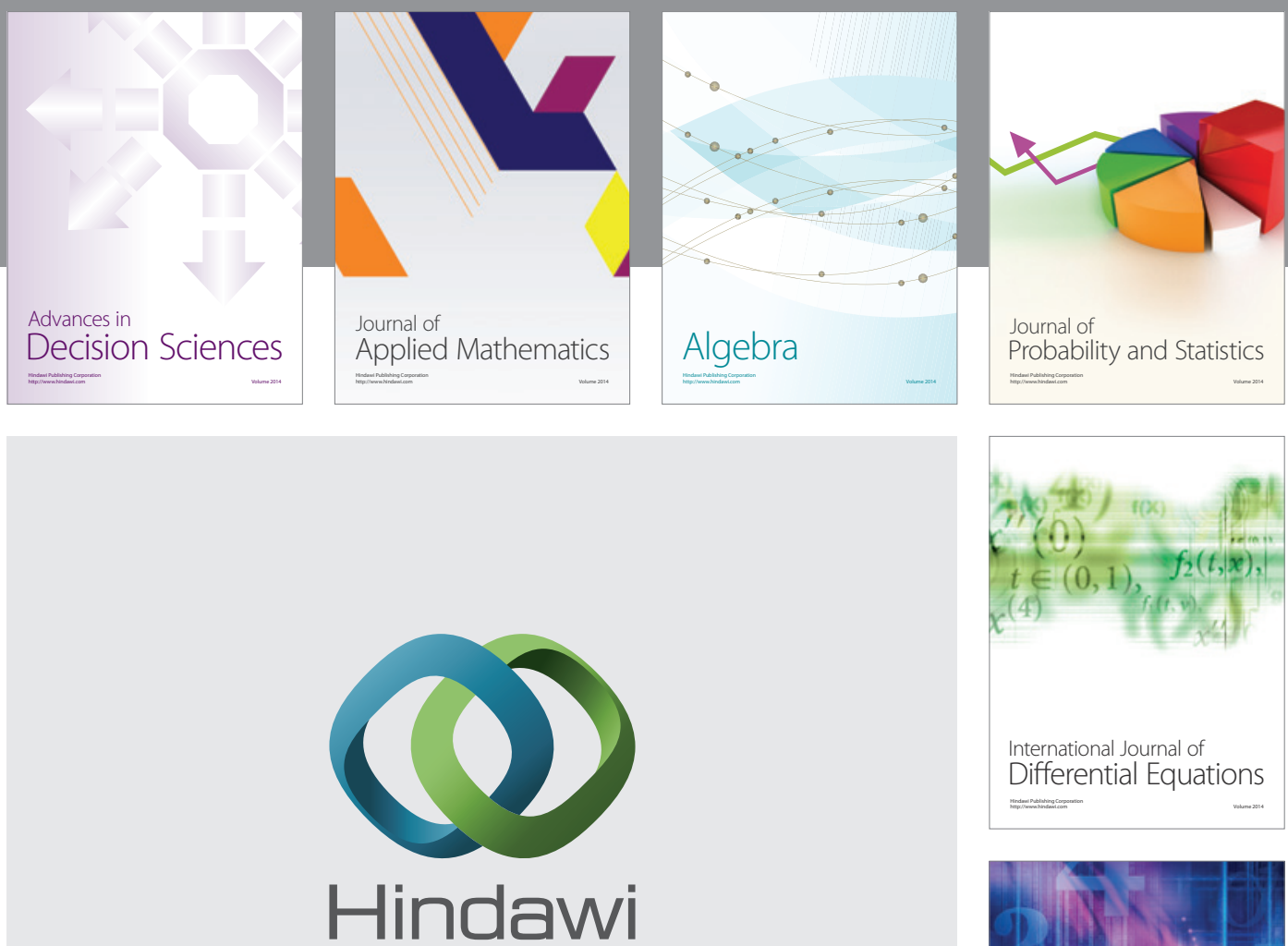

Submit your manuscripts at http://www.hindawi.com
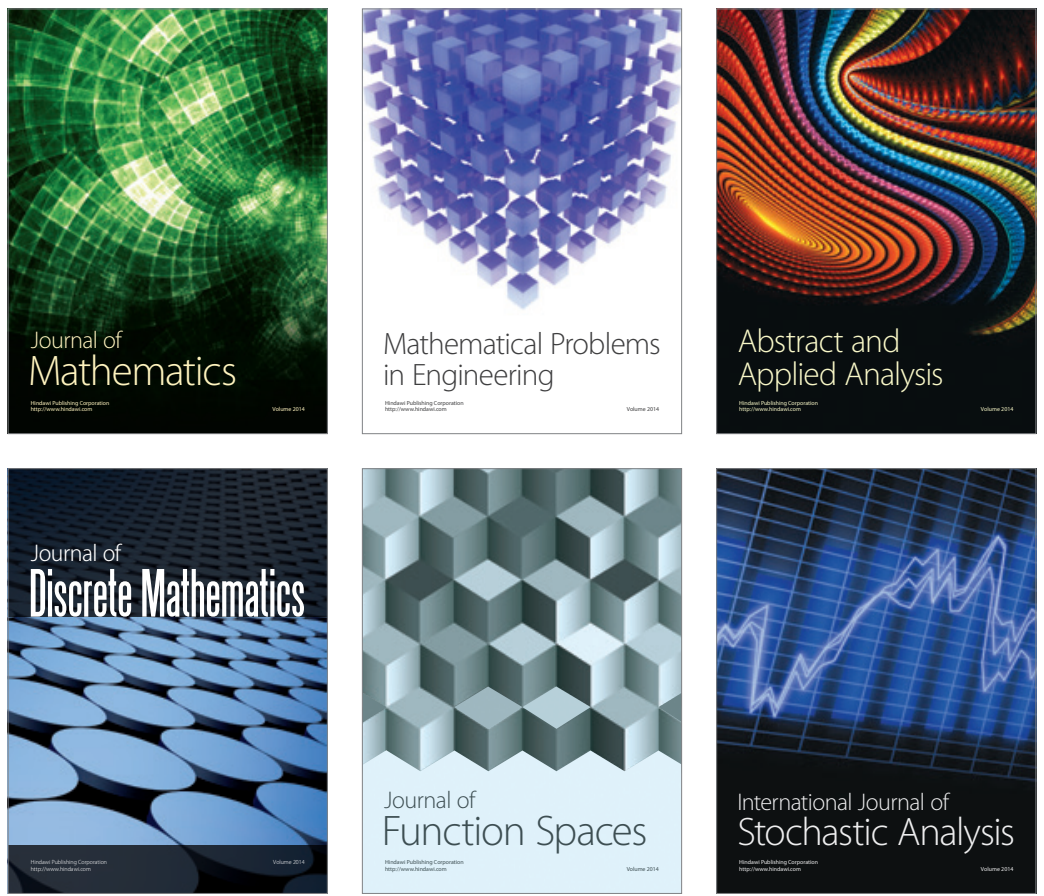

Journal of

Function Spaces

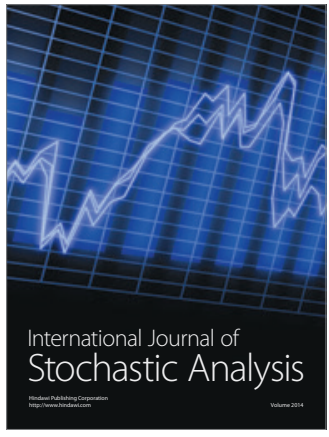

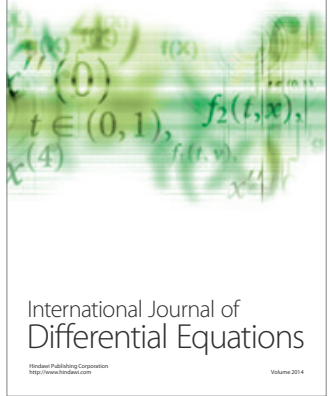
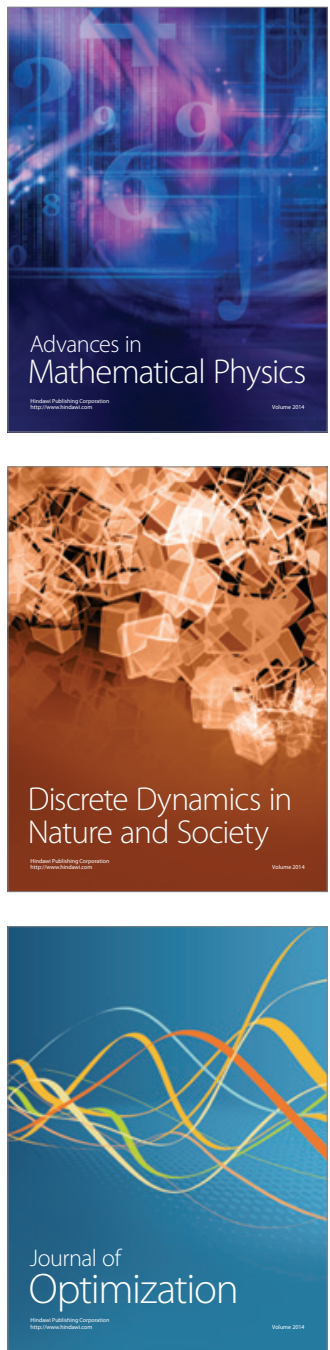